\title{
Hypersurfaces with parallel affine curvature tensor $R^{*}$
}

\author{
by BARBARA OpozDa (Kraków) and \\ LeOPOLd Verstraelen (Leuven)
}

\begin{abstract}
In $[\mathrm{OV}]$ we introduced an affine curvature tensor $R^{*}$. Using it we characterized some types of hypersurfaces in the affine space $\mathbb{R}^{n+1}$. In this paper we study hypersurfaces for which $R^{*}$ is parallel relative to the induced connection.
\end{abstract}

1. Let $M$ be an $n$-dimensional connected manifold and $f: M \rightarrow \mathbb{R}^{n+1}$ its immersion into the standard affine space $\mathbb{R}^{n+1}$. Denote by $D$ the standard connection in $\mathbb{R}^{n+1}$. If $\xi$ is an equiaffine transversal vector field for $f$, that is, $D \xi$ is tangential to $f$, then the formulas of Gauss and Weingarten can be written as follows:

$$
\begin{gathered}
D_{X} f_{*} Y=f_{*} \nabla_{X} Y+h(X, Y) \xi, \\
D_{X} \xi=-f_{*} S X,
\end{gathered}
$$

where $X, Y$ are tangent vector fields on $M, \nabla$ is the induced connection on $M, h$ the second fundamental form and $S$ the shape operator. It is known that the rank of $h$ is independent of the choice of a transversal vector field. If the rank is equal to $n$ everywhere on $M$, then $f$ is called nondegenerate. For a nondegenerate hypersurface there exists a unique (up to a constant) equiaffine transversal vector field such that

$$
\operatorname{tr}_{h}\left(\nabla_{X} h\right)(\cdot, \cdot)=0
$$

for every $X \in T M$. This transversal vector field is called the affine normal.

Throughout the paper we shall study nondegenerate hypersurfaces endowed with equiaffine transversal vector fields. The relationship between $\nabla$, $h$ and $S$ is given by the fundamental equations

$$
R(X, Y) Z=h(Y, Z) S X-h(X, Z) S Y \quad \text { (Gauss), }
$$

1991 Mathematics Subject Classification: Primary 53A15.

Key words and phrases: affine normal, induced connection, curvature tensor.

The first author was supported by the Catholic University of Leuven and the KBN grant 2 P03A 02016. 


$$
\begin{aligned}
\nabla h(X, Y, Z) & =\nabla h(Y, X, Z) & & (\text { Codazzi I), } \\
\nabla S(X, Y) & =\nabla S(Y, X) & & (\text { Codazzi II }), \\
h(S X, Y) & =h(X, S Y) & & (\text { Ricci }),
\end{aligned}
$$

where $R$ is the curvature tensor of $\nabla$. The tensor field $R^{*}$ given by $R^{*}(X, Y) Z$ $=R(X, Y) S Z$ is a curvature tensor relative to $h$, that is, $h\left(R^{*}(X, Y) Z, W\right)$ is skew-symmetric for $Z$ and $W$. Note that $R$ is not, in general, a curvature tensor relative to $h$.

One can study various conditions imposed on $R^{*}$. For instance, in [OV] we proved that $R_{x}^{*}=0$ if and only if $\operatorname{rk} S_{x} \leq 1$. If $R^{*}$ constantly vanishes on $M$, then $M=M_{1} \cup M_{2}$, where $M_{1}=\left\{x \in M: S_{x}=0\right\}$ and $M_{2}=$ $\left\{x \in M: \operatorname{rk} S_{x}=1\right\}$. A transversal vector field $\xi$ is a curve in $M_{2}$, that is, around each point of $M_{2}$ there is a coordinate system $\left(u_{1}, \ldots, u_{n}\right)$ such that $\xi$ depends only on one variable. Surfaces in $\mathbb{R}^{3}$ with affine normals which are curves are described in $[\mathrm{O}]$ and $[\mathrm{OS}]$. For instance, such surfaces with nondiagonalizable shape operator are characterized as follows:

TheOREM 1. Let $f: M \rightarrow \mathbb{R}^{3}$ be a nondegenerate surface with affine normal $\xi$. The following conditions are equivalent:

(a) $\xi$ is a curve and the affine shape operator $S$ is nondiagonalizable.

(b) $f$ is a minimal ruled surface.

(c) $f$ is a ruled surface with planar generators.

Surfaces with diagonalizable shape operator are characterized by differential equations. For instance, in the case of surfaces with parallel image of the shape operator we have

TheOREM 2. Let $f: M \rightarrow \mathbb{R}^{3}$ be a nondegenerate surface equipped with the affine normal $\xi$ inducing $\nabla, h$ and $S$. If $S$ is diagonalizable, im $S$ is 1-dimensional and parallel relative to $\nabla$, then for every $x \in M$ there is a coordinate system $(u, v)$ around $x$ and functions $\phi(u, v), a(u)$ such that $\phi$ is positive valued, $\phi$ and a satisfy the equation

and

$$
\varepsilon_{1} \phi_{u u}+\varepsilon_{1} \frac{a^{\prime}}{2} \phi_{u}+\varepsilon_{2} e^{-a} \phi_{v v}=-\phi
$$

$$
\begin{aligned}
\nabla_{\partial_{u}} \partial_{u}=\left((\log \phi)_{u}-\frac{a^{\prime}}{2}\right) \partial_{u}, & h\left(\partial_{u}, \partial_{u}\right)=\varepsilon_{1} \phi e^{-a}, \\
\nabla_{\partial_{u}} \partial_{v}=(\log \phi)_{v} \partial_{u}, & h\left(\partial_{u}, \partial_{v}\right)=0, \\
\nabla_{\partial_{v}} \partial_{v}=-\varepsilon(\log \phi)_{u} e^{a} \partial_{u}, & h\left(\partial_{v}, \partial_{v}\right)=\varepsilon_{2} \phi, \\
\quad S \partial_{u}=e^{a} \phi^{-1} \partial_{u}, & S \partial_{v}=0,
\end{aligned}
$$

where $\varepsilon_{1}, \varepsilon_{2}= \pm 1$ and $\varepsilon=\varepsilon_{1} \varepsilon_{2}$. The immersion $f$ is equal modulo the special affine group $S A(3, \mathbb{R})$ to 


$$
f(u, v)=p(v)+q(u, v)
$$

where $p(v)$ and $q(u, v)$ are obtained in the following way. Let $U=I \times J$ be a domain of a coordinate system $(u, v)$ and $\xi(u): I \rightarrow \mathbb{R}^{2}=\mathbb{R}^{2} \times\{0\} \subset \mathbb{R}^{3}$ be a centroaffine curve satisfying the equation

$$
\xi^{\prime \prime}=-\varepsilon_{1} \xi+\frac{a^{\prime}}{2} \xi^{\prime}
$$

Let $q(u, v)$ and $p(v)$ be arbitrary functions satisfying the equations

$$
q_{u}=-e^{-a} \phi \xi^{\prime}, \quad p^{\prime \prime}=-q_{v v}+\varepsilon \phi_{u} \xi^{\prime}+\varepsilon_{2} \phi \xi
$$

and the condition $p^{\prime}(v) \notin \mathbb{R}^{2}$ for every $v \in J$. The vector field $\xi(u, v)=\xi(u)$ is the affine normal for $f(u, v)$ up to a constant.

The case where $\operatorname{im} S$ is not $\nabla$-parallel is more complicated and we refer to [OS] for information about it.

In $[\mathrm{OV}]$ we also introduced the Ricci and Weyl tensors determined by $R^{*}$. We proved that for a quasi-umbilical hypersurface the Weyl tensor vanishes. The converse (for manifolfds of dimension greater than 3 ) is proved in $[D]_{1}$. In $[\mathrm{D}]_{1}$ and $[\mathrm{D}]_{2}$ other conditions on $R^{*}$ are studied.

2. In this paper we prove the following result.

THEOREM 3. Let $f: M \rightarrow \mathbb{R}^{n+1}$ be a nondegenerate hypersurface equipped with an equiaffine transversal vector field $\xi$ inducing a connection $\nabla$. If $\nabla R^{*}=0$, then $R^{*}$ constantly vanishes on $M$ or $f$ is a nondegenerate central quadric in $\mathbb{R}^{n+1}$ and $\xi$ is its affine normal (up to a constant).

Proof. If there exists a point $x \in M$ such that $R_{x}^{*}=0$, then $R^{*}=0$ on the whole $M$ because $R^{*}$ is parallel relative to a connection. From now on we assume that $R^{*} \neq 0$ everywhere on $M$. In this case $\operatorname{rk} S_{x}>1$ for every $x \in M$.

We first consider the condition $R \cdot R^{*}=0$. We have

$$
\begin{aligned}
\left(R(X, Y) \cdot R^{*}\right) & (Z, V) W \\
= & (h(V, S W) h(Y, S Z)-h(Z, S W) h(Y, S V)) S X \\
+ & (h(Z, S W) h(X, S V)-h(V, S W) h(X, S Z)) S Y \\
+ & (h(Y, V) h(Z, S W)-h(Y, Z) h(V, S W)) S^{2} X \\
+ & (h(X, Z) h(V, S W)-h(X, V) h(Z, S W)) S^{2} Y \\
+ & (h(X, V) h(S Y, S W)-h(Y, V) h(S X, S W) \\
& \left.+h(X, W) h\left(V, S^{2} Y\right)-h(Y, W) h\left(V, S^{2} X\right)\right) S Z \\
+ & (h(Y, Z) h(S X, S W)-h(X, Z) h(S Y, S W) \\
& \left.+h(Y, W) h\left(Z, S^{2} X\right)-h(X, W) h\left(Z, S^{2} Y\right)\right) S V .
\end{aligned}
$$

We consider a few cases. In all the cases $x$ denotes any point of $M$. 
Case I. Assume first that $S_{x}$ is diagonalizable. By Lemma 1 of [VV] we know that $S_{x}$ and $h_{x}$ are simultaneously diagonalizable. Let $e_{1}, \ldots, e_{n}$ be an $h$-orthonormal basis of $T_{x} M$ consisting of eigenvectors of $S_{x}$. Let $\varrho_{1}, \ldots, \varrho_{n}$ be the eigenvalues of $S_{x}$ corresponding to $e_{1}, \ldots, e_{n}$ respectively. If $\operatorname{dim} M=2$, then, by (2.1), we get

$$
0=\left(R\left(e_{1}, e_{2}\right) \cdot R^{*}\right)\left(e_{1}, e_{2}\right) e_{1}=\varrho_{1} \varrho_{2}\left(\varrho_{2}-\varrho_{1}\right) h\left(e_{1}, e_{1}\right) h\left(e_{2}, e_{2}\right) e_{1}
$$

and consequently $\varrho_{1}=\varrho_{2}$. If $\operatorname{dim} M>2$ and $i, j, k$ are mutually distinct, then $\left(R\left(e_{j}, e_{i}\right) \cdot R^{*}\right)\left(e_{k}, e_{i}\right) e_{k}=0$ yields

$$
\varrho_{k} \varrho_{j}\left(\varrho_{j}-\varrho_{i}\right)=0 .
$$

Since rk $S_{x}>1$, we can assume that $\varrho_{1}$ and $\varrho_{2}$ are not zero. If we put $\varrho_{k}=\varrho_{1}$ and $\varrho_{j}=\varrho_{2}$ in $(2.2)$, then we get $\varrho_{2}=\varrho_{i}$ for every $i \geq 3$. By taking $k=2$, $j=1$ and $i \geq 3$ we obtain $\varrho_{1}=\varrho_{i}$. Consequently, $\varrho_{1}, \ldots, \varrho_{n}$ are all equal, that is, $S_{x}=\varrho I_{x}$ for some nonzero $\varrho$ where $I_{x}$ is the identity endomorphism of $T_{x} M$.

In the next cases we assume that $S_{x}$ is not diagonalizable. In particular, $h_{x}$ is not definite.

CASE II. Assume that $\operatorname{dim} M=2$. Let $X, Y$ be an $h$-orthonormal basis of $T_{x} M$, i.e. $h(X, Y)=0, h(X, X)=1=-h(Y, Y)$. By (2.1) we get

$$
\begin{aligned}
0= & \left(R(X, Y) \cdot R^{*}\right)(X, Y) X \\
= & \left(h(Y, S X)^{2}-h(X, S X) h(Y, S Y)+h(S X, S X)+h(S Y, S Y)\right) S X \\
& -2 h(S X, S Y) S Y-h(X, S X) S^{2} X+h(Y, S X) S^{2} Y .
\end{aligned}
$$

Since $X, Y$ is an $h$-orthonormal basis, we obtain

$$
\begin{aligned}
0= & h\left(\left(R(X, Y) \cdot R^{*}\right)(X, Y) X, Y\right) \\
= & h(Y, S X)^{2} h(S X, Y)-h(X, S X) h(Y, S Y) h(S X, Y) \\
& +h(S X, X)^{2} h(S X, Y)-h(S X, Y)^{2} h(S X, Y) \\
& +h(S Y, X)^{2} h(S X, Y)-h(S Y, Y)^{2} h(S X, Y) \\
& -2 h(S X, X) h(S Y, X) h(S Y, Y)+2 h(S X, Y) h(S Y, Y)^{2} \\
& -h(X, S X)^{2} h(S Y, X)+h(X, S X) h(S X, Y) h(S Y, Y) \\
& +h\left((Y, S X) h(S Y, X)^{2}-h(Y, S X) h(S Y, Y)^{2}\right. \\
= & 2 h(X, S Y)\left(h(X, S Y)^{2}-h(S X, X) h(S Y, Y)\right) .
\end{aligned}
$$

Since $S_{x}$ is not diagonalizable, we have $h(S X, Y) \neq 0$. Thus

$$
h(X, S Y)^{2}-h(S X, X) h(Y, S Y)=0 .
$$

This means that $\operatorname{det} S_{x}=0$, which contradicts the assumption $\operatorname{rk} S_{x}>1$.

In cases III and IV the dimension of $M$ is assumed to be greater than 2 . 
CAse III. Assume that $\operatorname{rk} S_{x}<n$. We first show that $S_{x}^{2}=0$ on $T_{x} M$. By (2.1) applied to any $X, Y, Z, V \in T_{x} M$ and $0 \neq W \in \operatorname{ker} S_{x}$ we get

$$
\begin{aligned}
(h(X, W) h( & \left.\left.S^{2} V, Y\right)-h(Y, W) h\left(V, S^{2} X\right)\right) S Z \\
& +\left(h(Y, W) h\left(Z, S^{2} X\right)-h(X, W) h\left(Z, S^{2} Y\right)\right) S V=0,
\end{aligned}
$$

Let $V \in T_{x} M \backslash \operatorname{ker} S_{x}$. Since $\operatorname{rk} S_{x}>1$, there is $Z$ such that $S V, S Z$ are linearly independent. Then, by (2.3), we have

$$
h(X, W) h\left(S^{2} V, Y\right)=h(Y, W) h\left(V, S^{2} X\right)
$$

for every $X, Y$. It follows that

$$
h\left(S^{2} V, Y\right) W=h(Y, W) S^{2} V
$$

for every $Y \in T_{x} M$. Hence

$$
h\left(S^{2} V, Y\right)=0
$$

for every $Y \in\langle W\rangle^{\perp}$, where $\langle W\rangle^{\perp}$ is the subspace $h$-orthogonal to $W$. If there is a vector $W \in \operatorname{ker} S_{x}$ such that $h(W, W) \neq 0$, then the space $\langle W\rangle^{\perp}$ is an algebraic complement to $\operatorname{span}\{W\}$ in $T_{x} M$. Therefore, by (2.5) and the obvious fact $h\left(S^{2} V, W\right)=h(S V, S W)=0$, we get $S^{2}=0$ on $T_{x} M$. Assume now that $h(W, W)=0$ for every $W \in \operatorname{ker} S_{x}$. To get a contradiction we also assume that $S^{2}$ is not identically zero on $T_{x} M$. If there exist $W \in \operatorname{ker} S$ and $V$ such that $S^{2} V$ is not parallel to $W$, then (by (2.4)) $h(Y, W)=0$ for every $Y \in T_{x} M$, that is, $W=0$, which is a contradiction. Hence for every $V \notin \operatorname{ker} S$ and every $W \in \operatorname{ker} S$ the vector $S^{2} V$ is parallel to $W$. It follows that

$$
\operatorname{dim} \operatorname{ker} S_{x}=1
$$

and

$$
\operatorname{im} S_{x}^{2}=\operatorname{ker} S_{x}
$$

Assume that $n>3$. Let $\mathcal{L}$ be an algebraic complement to $\operatorname{ker} S_{x}$ in $T_{x} M$. Then $\operatorname{dim} \mathcal{L}=n-1$ and $S_{\mid \mathcal{L}}$ is an injection. Since rk $S_{x}=n-1$ and $n>3$, we have $\operatorname{dim}\left(\mathcal{L} \cap \operatorname{im} S_{x}\right) \geq 2$. Since $S_{x}$ restricted to $\mathcal{L} \cap \operatorname{im} S_{x}$ is an injection, we have $\mathrm{rk} S_{x}^{2} \geq 2$, which contradicts (2.6) and (2.7).

Assume $n=3$. Then $\operatorname{rk} S_{x}=2$. We now take $0 \neq X \in \operatorname{ker} S_{x}$. By (2.7) there is $Y$ such that $X=S^{2} Y$. We set $Z=S Y$ and choose $V$ such that $X=S Z$ and $S V$ are linearly independent. By (2.1) we get

$$
(h(X, Z) h(V, S W)+h(X, V) h(X, W)) X-2 h(X, Z) h(X, W) S V=0
$$

for every $W \in T_{x} M$. It follows that $h(X, Z)=0$, i.e. $Z \in\langle X\rangle^{\perp}$. Hence, by $(2.8), h(X, V) h(X, W)=0$ for every $W$, that is, $V \in\langle X\rangle^{\perp}$. Since $Z, V$ are linearly independent, they span $\langle X\rangle^{\perp}$. But $X \in\langle X\rangle^{\perp}$ and consequently $X=x_{1} Z+x_{2} V$ for some numbers $x_{1}, x_{2}$. Therefore $0=S X=x_{1} S Z+x_{2} S V$, 
i.e. $S Z, S V$ are linearly dependent, which is a contradiction. Consequently, $S^{2}=0$ on $T_{x} M$.

Let $Y, X$ be such that $S Y, S X$ are linearly independent. By (2.1) we have

$$
h(V, S W) h(Y, S Z)-h(Z, S W) h(Y, S V)=0
$$

for every $V, Z, W \in T_{x} M$. Since $\operatorname{dim}\langle Y\rangle^{\perp}=n-1$ and $\operatorname{rk} S_{x} \geq 2$, we have $\langle Y\rangle^{\perp} \cap \operatorname{im} S_{x} \neq\{0\}$. It follows that there is $Z$ such that $S Z \neq 0$ and $h(Y, S Z)=0$. For such a $Z$, by $(2.9)$, we get $h(S Z, W) h(Y, S V)=0$ for every $W$ and $V$, i.e. $h(S Y, V)=0$ for every $V$, contrary to $S Y \neq 0$.

CASE IV. Assume that $\operatorname{rk} S_{x}=n$. Since $n \geq 3$ and $S_{x}$ is an isomorphism, there are vectors $e_{1}, e_{2}, \widetilde{e}_{3} \in T_{x} M$ such that $e_{1}, e_{2}, S \widetilde{e}_{3}$ are $h$-orthonormal. We put $X=e_{2}, Y=\widetilde{e}_{3}, Z=e_{1}, V=e_{2}, W=\widetilde{e}_{3}$. By (2.1) we get

$$
h\left(e_{2}, e_{2}\right) h\left(S \widetilde{e}_{3}, S \widetilde{e}_{3}\right)=h\left(\widetilde{e}_{3}, \widetilde{e}_{3}\right) h\left(S e_{2}, S e_{2}\right)
$$

and

$$
\begin{aligned}
h\left(\widetilde{e}_{3}, e_{1}\right) h\left(S e_{2}, S \widetilde{e}_{3}\right)+h\left(\widetilde{e}_{3}, \widetilde{e}_{3}\right) h\left(e_{1}, S^{2} e_{2}\right) & \\
& -h\left(\widetilde{e}_{3}, e_{2}\right) h\left(e_{1}, S^{2} \widetilde{e}_{3}\right)=0 .
\end{aligned}
$$

Since $h\left(e_{2}, e_{2}\right) \neq 0$ and $h\left(S \widetilde{e}_{3}, S \widetilde{e}_{3}\right) \neq 0$, we obtain $h\left(\widetilde{e}_{3}, \widetilde{e}_{3}\right) \neq 0$. By $(2.10)$ and the fact that $h\left(e_{2}, e_{2}\right)= \pm 1$ we get

$$
h\left(e_{2}, e_{2}\right) h\left(S e_{2}, S e_{2}\right)=\frac{h\left(S \widetilde{e}_{3}, S \widetilde{e}_{3}\right)}{h\left(\widetilde{e}_{3}, \widetilde{e}_{3}\right)} .
$$

If we take $X=e_{1}, Y=\widetilde{e}_{3}, Z=e_{1}, V=e_{2}, W=e_{3}$, then (2.1) yields

$$
\begin{aligned}
-h\left(\widetilde{e}_{3}, e_{2}\right) h\left(S e_{1}, S \widetilde{e}_{3}\right)+h\left(e_{1}, \widetilde{e}_{3}\right) h\left(e_{2}, S^{2} \widetilde{e}_{3}\right) & \\
& -h\left(\widetilde{e}_{3}, \widetilde{e}_{3}\right) h\left(e_{2}, S^{2} e_{1}\right)=0
\end{aligned}
$$

and

$$
h\left(e_{1}, e_{1}\right) h\left(S e_{1}, S e_{1}\right)=\frac{h\left(S \widetilde{e}_{3}, S \widetilde{e}_{3}\right)}{h\left(\widetilde{e}_{3}, \widetilde{e}_{3}\right)} .
$$

Formulas (2.12) and (2.14) imply

$$
h\left(e_{1}, e_{1}\right) h\left(S e_{1}, S e_{1}\right)=h\left(e_{2}, e_{2}\right) h\left(S e_{2}, S e_{2}\right) .
$$

Since $e_{1}, e_{2}$ can be an arbitrary $h$-orthonormal pair, for any $h$-orthonormal basis $e_{1}, \ldots, e_{n}$ of $T_{x} M$ we have

$$
h\left(e_{i}, e_{i}\right) h\left(S^{2} e_{i}, e_{i}\right)=h\left(e_{j}, e_{j}\right) h\left(S^{2} e_{j}, e_{j}\right)
$$

for every $i, j=1, \ldots, n$. By comparing (2.11) and (2.13) we obtain

$$
h\left(\widetilde{e}_{3}, \widetilde{e}_{3}\right) h\left(S^{2} e_{1}, e_{2}\right)=0,
$$

that is,

$$
h\left(S^{2} e_{1}, e_{2}\right)=0 .
$$


Therefore, if $e_{1}, \ldots, e_{n}$ is an $h$-orthonormal basis of $T_{x} M$, then

$$
h\left(S^{2} e_{i}, e_{j}\right)=0
$$

for any $i \neq j, i, j=1, \ldots, n$. Formulas $(2.15),(2.17)$ imply that $S_{x}^{2}=\alpha I_{x}$. Of course $\alpha \neq 0$. Suppose $\alpha<0$. Then $S / \sqrt{-\alpha}$ is a complex structure on $T_{x} M$. In particular, $n$ is even. Hence $n \geq 4$. Let $e_{1}, \ldots, e_{n-1}, S \widetilde{e}_{n}$ be an $h$-orthonormal basis of $T_{x} M$ and let $X=e_{1}, Y=e_{2}, V=\widetilde{e}_{n}, W=e_{3}$ and $Z$ be such that $h\left(S e_{3}, Z\right) \neq 0$. Then

$$
\begin{aligned}
h\left(S^{2} Y, W\right) & =h\left(S^{2} X, W\right)=h(X, W)=h(Y, W) \\
& =h(S V, X)=h(S V, Y)=h(S V, W)=0 .
\end{aligned}
$$

Consequently, by $(2.1), h\left(e_{1}, \widetilde{e}_{n}\right) h\left(S e_{3}, Z\right)=0$, i.e. $h\left(e_{1}, \widetilde{e}_{n}\right)=0$. Since the order of $e_{1}, \ldots, e_{n-1}$ is not important, we have

$$
h\left(\widetilde{e}_{n}, e_{i}\right)=0
$$

for every $i=1, \ldots, n-1$, i.e. $S \widetilde{e}_{n}$ is parallel to $\widetilde{e}_{n}$. Let $S \widetilde{e}_{n}=\beta \widetilde{e}_{n}$. Then $S^{2} \widetilde{e}_{n}=\beta^{2} \widetilde{e}_{n}$. But $S^{2} \widetilde{e}_{n}=\alpha \widetilde{e}_{n}$ and $\alpha<0$, that is, we have a contradiction. Hence $\alpha>0$ and there are two complementary subspaces $T_{1}$ and $T_{2}$ of $T_{x} M$ such that $S_{x \mid T_{1}}=\sqrt{\alpha} I_{x}$ and $S_{x \mid T_{2}}=-\sqrt{\alpha} I_{x}$. In particular $S_{x}$ is diagonalizable, which is again a contradiction.

Summing up, for each $x \in M$ we have $S_{x}=\varrho I_{x}$. By the Codazzi equation $\varrho$ is constant on $M$. Since $R^{*} \neq 0, \varrho$ is not zero. Hence

$$
R^{*}(X, Y) Z=\varrho^{2}(h(Y, Z) X-h(X, Z) Y)
$$

and consequently

$$
\left(\nabla_{V} R^{*}\right)(X, Y) Z=\varrho^{2}(\nabla h(V, Y, Z) X-\nabla h(V, X, Z) Y) .
$$

Therefore $\nabla R^{*}=0$ implies that $\nabla h=0$ and, by a theorem of Berwald, $f: M \rightarrow \mathbb{R}^{n+1}$ is a quadratic hypersurface. The proof is complete.

\section{References}

$[\mathrm{D}]_{1} \quad$ R. Deszcz, Pseudosymmetry curvature conditions imposed on the shape operators of hypersurfaces in the affine space, Results Math. 20 (1991), 600-621.

$[\mathrm{D}]_{2}-$, Certain curvature characterizations of affine hypersurfaces, Colloq. Math. 63 (1992), 21-39.

[NS] K. Nomizu and T. Sasaki, Affine Differential Geometry, Cambridge Univ. Press, 1994.

[O] B. Opozda, A class of projectively flat surfaces, Math. Z. 219 (1995), 77-92.

[OS] B. Opozda and T. Sasaki, Surfaces whose images of affine normal are curves, Kyushu Math. J. 49 (1995), 1-10.

[OV] B. Opozda and L. Verstraelen, On a new curvature tensor in affine differential geometry, in: Geometry and Topology of Submanifolds II, World Sci., 1990, 271293. 
[VV] P. Verheyen and L. Verstraelen, Locally symmetric affine hypersurfaces, Proc. Amer. Math. Soc. 93 (1985), 101-105.

Institute of Mathematics Jagiellonian University

Reymonta 4

30-059 Kraków, Poland

E-mail: opozda@im.uj.edu.pl
Dept. Wiskunde Katholieke Universiteit Leuven Celestijnenlaan 200B B 3030 Leuven, Belgium E-mail: Leopold.Verstraelen@wis.kuleuven.ac.be

Reçu par la Rédaction le 15.12.1997

Révisé le 13.7.1998 